\title{
Vascular Access Vulnerability in Intensive Hemodialysis: A Significant Achilles' Heel?
}

\author{
Tom Cornelis $^{\mathrm{a}}$ Len A. Usvyat ${ }^{c, d}$ Jan H. Tordoir ${ }^{\mathrm{b}}$ Yuedong Wang \\ Michelle Wong $^{c}$ Karel M. Leunissen ${ }^{a}$ Frank M. van der Sande ${ }^{a}$ \\ Peter Kotanko ${ }^{c}$ Jeroen P. Kooman ${ }^{\mathrm{a}}$ \\ ${ }^{a}$ Division of Nephrology, Department of Internal Medicine, and ${ }^{b}$ Department of Surgery, Maastricht University \\ Medical Center, Maastricht, The Netherlands; ${ }^{C}$ Renal Research Institute, New York, N.Y., dFresenius Medical Care \\ North America, Waltham, Mass., and e University of California - Santa Barbara, Santa Barbara, Calif., USA
}

\section{Key Words}

Hemodialysis · Vascular access $\cdot$ Complications

\begin{abstract}
Background: Frequent hemodialysis (HD) may be associated with an increased risk of vascular access complications. Studies addressing vascular access outcomes in frequent HD show conflicting results. Methods: We searched Medline for trials looking at vascular access outcomes in frequent HD. Results: Nineteen studies met the inclusion criteria; only studies with a control group were included for analysis $(n=$ 15). The vascular access event rate was higher in intensive $\mathrm{HD}$ as compared to conventional HD (difference of 6.7 events per 100 patient-years, $p=0.009$ ). Overall event rates were not significantly different between conventional and intensive HD when stratified for access type, but were notably higher in the arteriovenous grafts and catheter group as compared to the arteriovenous fistula (AVF) group. Conclusion: Intensive HD is associated with an increased risk of vascular access complications. Overall reported event rates were lower in the AVF group. Further controlled studies should investigate whether a 'fistula first' strategy may be recommended also for intensive HD. @ $\quad$ 2014 S. Karger AG, Basel
\end{abstract}

\section{KARGER}

E-Mail karger@karger.com

www.karger.com/bpu

\section{Introduction}

Hemodialysis (HD) was performed routinely at home in the 1970s. Thereafter, for logistic and economic reasons, in-center conventional HD became the standard of care. In the 1990s, home HD underwent a significant revival under the impulse of Uldall et al. [1] from Toronto, who reintroduced home programs especially because of ample clinical benefits and reduced costs. Initially, home HD was performed with a dialysis catheter as vascular access. The question arose whether the use of arteriovenous fistulas (AVF) would be a potential barrier for home HD, especially in frequent HD.

Compared with conventional dialysis modalities, intensive HD (i.e. extended and/or frequent HD, either as short daily or nocturnal HD) is associated with multiple clinical benefits, including improved survival, quality of life, blood pressure control, left ventricular hypertrophy, better phosphate control, less sleep abnormalities, and improved fertility and pregnancy outcomes [2]. For these reasons intensive HD has been put forward as the optimal dialysis modality for patients with end-stage renal disease. In that vein, we previously recommended that the concept of 'intensive HD first' should be an obligatory
C 2014 S. Karger AG, Basel

0253-5068/14/0373-0222\$39.50/0 
part of predialysis patient education [3]. However, multiple barriers impede and even prevent the implementation of this strategy. One of the barriers is the creation and maintenance of proper vascular access. If intensive HD would lead to more frequent access events, it might result in increased morbidity, reduced quality of life, and increased resource utilization. In one of the early studies of that topic, Woods et al. [4] reported in 1999 that the number of access events decreased after conversion from conventional HD to daily HD. This finding was corroborated 1 year later by Quintaliani et al. [5]. In fact, these two studies were anticipated 30 years earlier by DePalma et al. [6], who stated in 1969 that previous shunt failures should be an indication for daily HD; this statement was made in the context of lower ultrafiltration rates and thus reduced rates of intradialytic hypotension and shunt thrombosis. However, more recent studies, including the Frequent Hemodialysis Network (FHN) trials, have cast doubt on this optimistic view. In the daily FHN trial, patients randomized to intensive HD experienced significantly more arteriovenous access repairs than patients randomized to conventional HD. Although nonsignificant, comparable trends were observed in the nocturnal FHN trial [7].

This somewhat ambiguous situation motivated us to review existing trials reporting vascular access outcomes in patients on intensive HD. Recently, a meta-analysis was performed and published by the Canadian Society of Nephrology to inform clinical practice guidelines for the provision of intensive HD [8]. In our review and analysis, we focused on access-related hospital admissions, access dysfunction, permanent access failure, and access infections in intensive and conventional HD patients.

\section{Materials and Methods}

\section{Research Questions}

The primary questions addressed in this review were: (1) what is the published experience with vascular access in intensive HD (short daily HD and/or nocturnal HD), (2) is there sufficient evidence to conclude that intensive HD is associated with an increased risk of vascular access complications, and (3) do vascular access complications differ between intensive and conventional $\mathrm{HD}$ with respect to access type [AVF, arteriovenous grafts (AVG), catheters]?

\section{Included Studies}

We included published full-text case series, cohort studies, and randomized controlled trials whenever two investigators independently agreed that the articles reported demographics and access events during a follow-up of 1 month or more in 5 or more adult patients (at least 18 years of age) receiving short daily HD or nocturnal HD. We included both home and in-center intensive HD studies. Only studies with a control group (parallel and/or prepost) were included for the analysis. Reports published in abstract form only were excluded.

\section{Finding Relevant Studies}

An independent review of citations from Medline (OVID 1966 to April 1, 2013) bibliographic database was conducted by two investigators. The search strategy made use of multiple synonyms for the terms dialysis, daily, frequent, intensive, vascular access, dysfunction, infections, hospitalizations, and outcomes.

\section{Statistical Analysis}

Studies were analyzed in a combined fashion by computing the total number of events and number of access-years per study. Separate analyses were performed by access type. Log-linear mixedeffects models for Poisson data with the offset of access-years and study-specific random effects were used to determine differences in event rates between intensive and conventional HD. Log-linear mixed-effects models with study-specific random effects were also used to assess the association between access events, access types, and kind of access dysfunction. Analyses were performed in SAS 9.3 (Cary, N.C., USA).

\section{Results}

\section{Description of Studies}

Nineteen studies met the inclusion criteria $[4,5,7$, 9-25]. Three studies were excluded because the vascular access event rates were not reported $[26,27]$ or because of missing demographic data [28]. Three studies were randomized controlled trials, 11 were prospective cohort studies, and 5 were retrospective cohort studies. Four studies had no control group $[9-11,18]$ and were not included in the analysis. Table 1 summarizes the demographic and treatment characteristics, including weekly dialysis frequency, treatment duration, location (home, in-center), mean or median time on intensive HD, and access type. Mean patient ages ranged from 35.6 to 59.9 years, and the majority of patients were male (range: 39.4-94\%). Mean conventional HD vintages ranged from 2.0 to 9.6 years.

\section{Vascular Access Complications in Intensive versus Conventional HD}

Table 2 summarizes vascular access event rates in intensive and conventional HD programs. Definitions and outcomes varied considerably between studies. To facilitate description of the results, we defined 'access dysfunction' as any intervention intended to salvage the access, and 'permanent access failure' as any event necessitating placement of a new vascular access. The access type 'access unknown' refers to studies where the access type was 
Table 1. Demographic and treatment characteristics reported in the included studies of IHD

\begin{tabular}{|c|c|c|c|c|c|c|c|c|c|c|c|c|c|}
\hline Ref. & Year & $\begin{array}{l}\text { Study } \\
\text { design }\end{array}$ & Control group & $\mathrm{n}$ & $\begin{array}{l}\text { Weekly } \\
\text { frequency, } \\
\text { days }\end{array}$ & $\begin{array}{l}\mathrm{HD} \\
\text { duration, } \\
\text { min }\end{array}$ & $\begin{array}{l}\text { Home/ } \\
\text { center, \% }\end{array}$ & $\begin{array}{l}\text { Average } \\
\text { age, } \\
\text { years }\end{array}$ & $\begin{array}{l}\text { Men, } \\
\%\end{array}$ & $\begin{array}{l}\text { IHD } \\
\text { vintage, } \\
\text { years }\end{array}$ & $\begin{array}{l}\text { AVF, } \\
\%\end{array}$ & $\begin{array}{l}\text { AVG, } \\
\%\end{array}$ & $\begin{array}{l}\text { Cathe- } \\
\text { ter, } \%\end{array}$ \\
\hline [9] & 1998 & PCS & no & 22 & $6-7$ & $90-120$ & $50 / 50$ & 52 & 50 & 4.8 & 100 & 0.0 & 0.0 \\
\hline [10] & 1998 & PCS & no & 12 & $6-7$ & $480-600$ & $100 / 0$ & $40 \pm 10$ & 66.6 & 1.3 & 0.0 & 0.0 & 100 \\
\hline$[4]$ & 1999 & PCS & pre-post & 72 & 6 (median) & 90 (median) & $58 / 42$ & 47 (median) & 74 & 2 (median) & 94.1 & 4.4 & 1.5 \\
\hline$[5]$ & 2000 & RCS & parallel & 24 & $6-7$ & 120 & $70 / 30$ & $47 \pm 13$ & 88 & 3.8 & 100 & 0 & 0 \\
\hline [11] & 2003 & PCS & no & 23 & 5.3 & 131 & $100 / 0$ & 51 & 69.6 & 1.5 & 61 & 21.7 & 17.3 \\
\hline [12] & 2003 & PCS & parallel & 11 & $5-6$ & $90-150$ & $100 / 0$ & 45.3 & 63.6 & $\begin{array}{l}1.6 \text { (5-36 } \\
\text { months) }\end{array}$ & 72.7 & 9.1 & 18.2 \\
\hline [12] & 2003 & PCS & parallel & 12 & $5-6$ & $360-480$ & $100 / 0$ & 44.2 & 76.9 & $\begin{array}{l}1.6(5-36 \\
\text { months })\end{array}$ & 53.8 & 15.4 & 30.8 \\
\hline [13] & 2003 & PCS & pre-post & 42 & $4-6$ & 114 & $7 / 93$ & 59.9 & 67 & 1.5 & 16 & 52 & 32 \\
\hline [14] & 2004 & RCS & pre-post & 17 & 6 & $120-150$ & $41 / 59$ & $46 \pm 13$ & 94 & $3.3 \pm 2$ & 100 & 0 & 0 \\
\hline [15] & 2004 & PCS & pre-post and parallel & $25-28$ & $5-6$ & $120-180$ & $50 / 50$ & $46 \pm 11$ & 80 & 1.3 and 1.8 & 78.6 & 21.4 & 0 \\
\hline [16] & 2004 & PCS & pre-post & 20 & 6 & $116 \pm 17$ & $0 / 100$ & $57 \pm 13$ & 80 & 4 weeks & 25 & 75 & 0 \\
\hline [17] & 2004 & PCS & pre-post & 12 & 6 & $75-150$ & $0 / 100$ & $56.0 \pm 8.6$ & 60 & 1.13 (mean) & NR & NR & NR \\
\hline [18] & 2006 & PCS & no & 26 & 6 & 150 & $0 / 100$ & $35.6 \pm 14.7$ & 61.5 & $2.80 \pm 1.54$ & 88.5 & 7.7 & 3.8 \\
\hline [19] & 2006 & RCS & pre-post and parallel & 33 & $5-6$ & $360-480$ & $100 / 0$ & 39 (median) & 39.4 & 3.7 (median) & 100 & 0 & 0 \\
\hline [20] & 2007 & RCT & parallel & 26 & $5-6$ & $>360$ & $100 / 0$ & 55.1 & 69 & 0.5 & 58 & 15 & 27 \\
\hline [21] & 2009 & RCS & parallel & 43 & 5.4 & 174 & $100 / 0$ & 40.9 & 72.1 & 2.7 (median) & NR & NR & NR \\
\hline [21] & 2009 & RCS & parallel & 94 & 5.7 & 450 & $100 / 0$ & 47 & 64.9 & 2.7 (median) & NR & NR & NR \\
\hline [22] & 2010 & RCS & parallel & 63 & 3.5 (median) & 540 (median) & $100 / 0$ & 51.7 & 79.4 & 1.9 & 81 & 19 & 0 \\
\hline$[7,23]$ & 2010 & RCT & parallel & 125 & 5.17 & 154 & $0 / 100$ & 48.9 & 62.4 & 1 & 66 & 18 & 17 \\
\hline$[7,24]$ & 2011 & RCT & parallel & 45 & 5.06 & 379 & $100 / 0$ & 51.7 & 64.4 & 1 & 49 & 7 & 44 \\
\hline [25] & 2012 & PCS & parallel & 26 & 6 & 180 & $0 / 100$ & $51 \pm 11.7$ & 65 & 4 & 60 & 36 & 4 \\
\hline
\end{tabular}

RCS = Retrospective cohort study; PCS = prospective cohort study; RCT = randomized controlled trial; IHD = intensive HD; NR = not reported.

not specified, whereas the access types 'AVF', 'AVG', and 'catheter' show results for studies looking at AVF, AVG, or catheter only, respectively. Table 2 shows the event rates of access-related hospital admissions, access dysfunction, permanent access failure, and access infections by access types. Differences in vascular access event rates between intensive and conventional HD and the respective $\mathrm{p}$ values are reported.

Our analysis, consolidating a total number of $1,540 \mathrm{ac}-$ cess-years for intensive HD and a total number of 2,284 access-years for conventional HD, indicates that across all access groups and access complications, intensive HD is associated with a higher vascular access event rate as compared with conventional HD (difference $=6.7$ events per 100 patient-years, $\mathrm{p}=0.009$ for all included studies; fig. 1). Overall event rates in the AVG group (61.0 events per 100 patient-years in conventional HD and 98.6 events per 100 patient-years in intensive HD) and catheter group (36.0 events per 100 patient-years in conventional HD and 52.9 events per 100 patient-years in intensive HD) were higher than in the AVF group. The vascular access event rates were also higher, though not significant, in patients undergoing intensive HD compared to conventional HD for both the AVG group (difference = 37.6 events per 100 patient-years, $\mathrm{p}=0.139)$ and catheter group (difference $=16.9$ events per 100 patient-years, $\mathrm{p}=$ 0.133 ); the difference in the AVF group was smaller (difference $2.7, p=0.162$ ).

\section{Discussion}

Our review, which comprehensively summarizes the currently published literature on vascular access in intensive HD, suggests an increased risk of vascular access complications in intensive HD as compared to conventional HD. Although the difference in vascular access complications between intensive and conventional HD in our analysis is rather small from a clinical point of view, this finding may have important implications for the adoption of intensive HD, such as an increased need for vascular access vigilance and interventions.

No significant difference in overall vascular access complications was found between intensive and conventional HD looking at patients with AVF, AVG, or catheter separately. However, a trend towards more pronounced differences between intensive and conventional HD was observed in the AVG and catheter group as compared to 
Table 2. Vascular access event rates in intensive and conventional HD

\begin{tabular}{|c|c|c|c|c|c|c|c|c|}
\hline \multirow{2}{*}{$\begin{array}{l}\text { Access } \\
\text { complication }\end{array}$} & \multirow[t]{2}{*}{ Access type } & \multirow[t]{2}{*}{ Study } & \multicolumn{2}{|l|}{ Intensive HD } & \multicolumn{2}{|c|}{ Conventional HD } & \multirow[t]{2}{*}{$\mathrm{RR}$} & \multirow[t]{2}{*}{$\mathrm{p}$} \\
\hline & & & $\mathrm{FU}^{\mathrm{a}}$ & event rate ${ }^{b}$ & $\mathrm{FU}^{\mathrm{a}}$ & event rate ${ }^{b}$ & & \\
\hline \multirow{24}{*}{$\begin{array}{l}\text { Access } \\
\text { dysfunction }\end{array}$} & \multirow{7}{*}{$\begin{array}{l}\text { all accesses } \\
\text { combined }\end{array}$} & {$[4]$} & 238.6 & 5.0 & 50.5 & 28 & 0.18 & $<0.001$ \\
\hline & & {$[15]$} & 50.9 & 28 & 103.3 & 34 & 0.8 & NS \\
\hline & & {$[16]$} & 1.5 & 130 & 1.5 & 0 & & NS \\
\hline & & {$[17]$} & 11.5 & 160 & 11.5 & 150 & 1.1 & 0.807 \\
\hline & & {$[20]$} & 13 & 138 & 12.5 & 144 & 0.96 & NR \\
\hline & & {$[22]$} & 86.2 & 50 & 142.7 & 49 & 1.02 & NS \\
\hline & & {$[25]$} & 71 & $40(27-57)$ & 129 & $54(43-67)$ & 0.74 & 0.33 \\
\hline & \multirow[t]{6}{*}{ AVF } & {$[11]$} & 21.1 & 52 & none & & & \\
\hline & & {$[12]$} & NR & $52 \pm 147$ & NR & $18 \pm 52$ & 2.9 & NS \\
\hline & & {$[13]$} & 15.3 & 59 & 90 & 27 & 2.2 & NR \\
\hline & & {$[14]$} & $21(\operatorname{Acc} Y)$ & 0.6 & $21(\operatorname{Acc} Y)$ & 14.6 & 0.04 & NS \\
\hline & & {$[7,23]$} & 76.9 & 35 & 67.1 & 28 & 1.25 & NR \\
\hline & & {$[7,24]$} & 22 & 64 & 18.8 & 21 & 3.05 & NR \\
\hline & \multirow[t]{5}{*}{ AVG } & [11] & 8.75 & 137 & none & & & \\
\hline & & {$[12]$} & NR & $158 \pm 179$ & NR & $212 \pm 195$ & 0.7 & NS \\
\hline & & [13] & 44.9 & 116 & 24.7 & 113 & 1 & NR \\
\hline & & {$[7,23]$} & 18.9 & 206 & 20.8 & 91 & 2.26 & NR \\
\hline & & {$[7,24]$} & 2.3 & 87 & 5.4 & 56 & 1.55 & NR \\
\hline & \multirow[t]{6}{*}{ catheter } & {$[10]$} & 15.5 & 77 & none & & & \\
\hline & & [11] & 4.2 & 144 & none & & & \\
\hline & & {$[12]$} & NR & $373 \pm 442$ & NR & $451 \pm 696$ & 0.8 & NS \\
\hline & & {$[13]$} & 8.8 & 46 & 5.2 & 153 & 0.3 & NR \\
\hline & & {$[7,23]$} & 18.9 & 21 & 20.8 & 5 & 4.2 & NR \\
\hline & & {$[7,24]$} & 18.1 & 06 & 19 & 5 & 1.2 & NR \\
\hline \multirow{13}{*}{$\begin{array}{l}\text { Permanent } \\
\text { access failure }\end{array}$} & \multirow{4}{*}{$\begin{array}{l}\text { all accesses } \\
\text { combined }\end{array}$} & [11] & 31.3 & 12 & none & & & \\
\hline & & {$[15]$} & 50.9 & 16 & 103.3 & 14 & 1.1 & NR \\
\hline & & [18] & 72.7 & 40 & none & & & \\
\hline & & {$[22]$} & 86.2 & $7(3-15)$ & 142.7 & $5(2-10)$ & 1.4 & NS \\
\hline & \multirow[t]{4}{*}{ AVF } & [9] & 106.3 & 2 & none & & & \\
\hline & & [5] & $91.2^{\mathrm{c}}$ & 2 & $421.6^{c}$ & 10 & 0.2 & $<0.01$ \\
\hline & & {$[7,23]$} & 76.9 & 10 & 67.1 & 12 & 0.83 & NR \\
\hline & & {$[7,24]$} & 22 & 18 & 18.8 & 5 & 3.6 & NR \\
\hline & \multirow[t]{2}{*}{ AVG } & {$[7,23]$} & 18.9 & 63 & 20.8 & 34 & 1.85 & NR \\
\hline & & {$[7,24]$} & 2.3 & 0 & 5.4 & 56 & & NR \\
\hline & \multirow[t]{3}{*}{ catheter } & [19] & $13.1(\mathrm{CVCY})$ & 213 & 12.7 (CVCY) & 107 & 1.99 & 0.03 \\
\hline & & {$[7,23]$} & 18.9 & 48 & 20.8 & 43 & 1.12 & NR \\
\hline & & {$[7,24]$} & 18.1 & 72 & 19 & 63 & 1.14 & NR \\
\hline Access & all accesses & [21] (SDHD) & $306.4^{\mathrm{c}}$ & 10 & $2,906.1^{c}$ & 20 & 0.5 & 0.52 \\
\hline admissions & combined & [21] (NHD) & $441^{\mathrm{c}}$ & 8 & $4,136^{\mathrm{c}}$ & 5 & 1.6 & 0.49 \\
\hline & & {$[7,23]$} & 121.2 & 25 & 127.8 & 19 & 1.32 (HR 0.99) & 0.97 (HR) \\
\hline & & {$[7,24]$} & 39.5 & 20 & 47.1 & 8 & $2.5($ HR 2.15$)$ & 0.20 (HR) \\
\hline & catheter & {$[10]$} & 15.5 & 6 & none & & & \\
\hline & & [19] & 13.1 (CVCY) & 15 & 12.7 (CVCY) & 0 & & 0.26 \\
\hline Access & all accesses & {$[22]$} & 86.2 & 16 & 142.7 & 7 & 2.29 & NR \\
\hline infections & combined & {$[20]$} & 13 & 38 & 12.5 & 32 & 1.19 & NR \\
\hline & AVF & {$[7,23]$} & 76.9 & 0 & 67.1 & 0 & & NR \\
\hline & & {$[7,24]$} & 22 & 0 & 18.8 & 0 & & NR \\
\hline & AVG & {$[7,23]$} & 18.9 & 11 & 20.8 & 10 & 1.1 & NR \\
\hline & & {$[7,24]$} & 2.3 & 0 & 5.4 & 19 & & NR \\
\hline & catheter & {$[10]$} & 15.5 & 65 & none & & & \\
\hline & & {$[12]$} & NR & $5.2 \pm 114$ & NR & $252 \pm 453$ & 0.02 & 0.081 \\
\hline & & {$[19]$} & 13.1 (CVCY) & 84 & 12.7 (CVCY) & 63 & 1.33 & NR \\
\hline & & {$[7,23]$} & 18.9 & 37 & 20.8 & 10 & 3.7 & NR \\
\hline & & {$[7,24]$} & 18.1 & 28 & 19 & 21 & 1.33 & NR \\
\hline
\end{tabular}

$\mathrm{FU}=$ Follow-up; RR = relative risk (intensive vs. control); NS = not significant; $\mathrm{NR}=$ not reported; CVCY = central venous catheter-years; SDHD = short daily HD; NHD = nocturnal HD; Acc Y = access-years. Access infections: [22] = total septic access events; [20] = bacteremia; [7, 10, 23, $24]$ = loss due to infection; [19] = bacteremia and exit/tunnel infection; [12] = not specified. ${ }^{\mathrm{a}}$ In patient-years. ${ }^{\mathrm{b}}$ In number of events per 100 patientyears. ${ }^{c}$ Calculated from number of patients and mean number of months on dialysis/follow-up. 


\begin{tabular}{|c|c|c|c|c|c|}
\hline & \multirow{2}{*}{$\begin{array}{l}\text { Access- } \\
\text { years }\end{array}$} & \multirow[t]{2}{*}{ Relative risk (intensive/conventional) } & \multicolumn{3}{|l|}{ Event rate } \\
\hline & & & difference & ratio & $\mathrm{p}$ \\
\hline $\begin{array}{l}\text { Access admissions } \\
\text { Access dysfunction } \\
\text { Access infection } \\
\text { Permanent access failure } \\
\text { AVF (all) }\end{array}$ & $\begin{array}{r}0 \\
332 \\
185 \\
228 \\
744\end{array}$ & 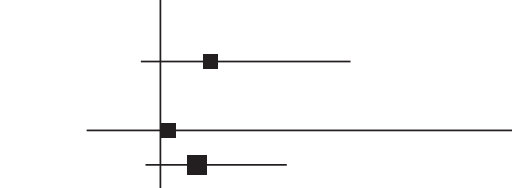 & $\begin{array}{l}0.116 \\
0.000 \\
0.008 \\
0.027\end{array}$ & $\begin{array}{l}1.545 \\
1.077 \\
1.391\end{array}$ & $\begin{array}{l}0.137 \\
0.872 \\
0.162\end{array}$ \\
\hline $\begin{array}{l}\text { Access admissions } \\
\text { Access dysfunction } \\
\text { Access infection } \\
\text { Permanent access failure } \\
\text { AVG (all) }\end{array}$ & $\begin{array}{r}0 \\
117 \\
47 \\
47 \\
212\end{array}$ & $\longrightarrow$ & $\begin{array}{r}0.429 \\
-0.019 \\
0.176 \\
0.376\end{array}$ & $\begin{array}{l}1.456 \\
0.835 \\
1.449 \\
1.478\end{array}$ & $\begin{array}{l}0.171 \\
0.874 \\
0.547 \\
0.139\end{array}$ \\
\hline $\begin{array}{l}\text { Access admissions } \\
\text { Access dysfunction } \\
\text { Access infection } \\
\text { Permanent access failure } \\
\text { Catheter (all) }\end{array}$ & $\begin{array}{r}26 \\
91 \\
103 \\
103 \\
322\end{array}$ & $\longrightarrow$ & $\begin{array}{r}0.153 \\
-0.024 \\
0.193 \\
0.341 \\
0.169\end{array}$ & $\begin{array}{l}0.696 \\
1.683 \\
1.483 \\
1.419\end{array}$ & $\begin{array}{l}0.516 \\
0.264 \\
0.217 \\
0.133\end{array}$ \\
\hline $\begin{array}{l}\text { Access admissions } \\
\text { Access dysfunction } \\
\text { Access infection } \\
\text { Permanent access failure } \\
\text { Access unknown (all) }\end{array}$ & $\begin{array}{r}985 \\
924 \\
254 \\
383 \\
2,546\end{array}$ & 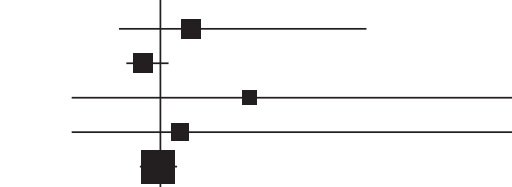 & $\begin{array}{r}0.073 \\
-0.209 \\
0.098 \\
0.015 \\
0.009\end{array}$ & $\begin{array}{l}1.324 \\
0.811 \\
1.975 \\
1.213 \\
0.965\end{array}$ & $\begin{array}{l}0.311 \\
0.112 \\
0.307 \\
0.673 \\
0.702\end{array}$ \\
\hline $\begin{array}{l}\text { Access admissions } \\
\text { Access dysfunction } \\
\text { Access infection } \\
\text { Permanent access failure } \\
\text { All }\end{array}$ & $\begin{array}{r}1,011 \\
1,464 \\
589 \\
761 \\
3,824\end{array}$ & - & $\begin{array}{r}0.073 \\
-0.049 \\
0.065 \\
0.103 \\
0.067\end{array}$ & $\begin{array}{l}1.350 \\
1.144 \\
1.633 \\
1.307 \\
1.224\end{array}$ & $\begin{array}{l}0.237 \\
0.134 \\
0.106 \\
0.150 \\
0.009\end{array}$ \\
\hline & & $\begin{array}{lll}1 & 1 & \\
2 & 3 & 4\end{array}$ & 5 & & \\
\hline
\end{tabular}

Fig. 1. Forest plot of vascular access event rates for all accesses in intensive as compared to conventional HD; analysis of studies with a control group only $(\mathrm{n}=15)$. There was a statistically significantly higher vascular access event rate in intensive HD as compared to conventional HD looking at all sorts of access complications in all access types together (difference of 0.067 events per patientyear, relative risk $=1.224, \mathrm{p}=0.009$ ). No difference in vascular access complications between intensive and conventional HD in the different access types could be demonstrated. the AVF group. In addition, the reported vascular access event rates in the AVG and catheter group were higher as compared to native AVF, a finding well established in many previous studies and reflected in international and national guidelines [29]. AVG may be especially susceptible to early failure in intensive HD because more frequent cannulation may promote weakening of the graft. On the other hand, in patients treated with intensive HD and catheters as vascular access, the need for more frequent manipulation may increase the risk of catheter-related infections. In line with current recommendations for conventional $\mathrm{HD}$, one may be tempted to interpret these results as support for the 'fistula first' strategy advocating that AVF should be the vascular access of first choice, also in patients treated with intensive HD. It should be acknowledged, however, that in no study was a randomized head-on comparison between different access types performed [30].

Despite the repeated cannulation-induced fistula trau$\mathrm{ma}$, the multifactorial pathobiology of access failure may offer an explanation for the relatively low vascular access event rate of AVF in intensive HD. Venous outflow obstruction plays an important role in vascular access failure, and neointimal hyperplasia by vascular smooth muscle and hyperplastic myofibroblasts is central in the genesis of venous outflow stenosis [31-35]. Repeated cannulation of the access triggers the local release of local cytokines which have been implicated in neointimal hy- 
perplasia [36]. However, poor nutrition, inflammation, atherosclerosis, hemodynamic instability, and uremic toxin levels also play a role in vascular access failure [3739]. Intensive HD may be the best currently available HD modality to protect the vascular access against these potentially damaging factors [2]. Furthermore, hyperhomocysteinemia is associated with vascular access thrombosis in dialysis patients [40]; it was also demonstrated that homocysteine levels are significantly reduced in nocturnal HD patients, compared to conventional HD patients [41]. These potential protective effects of intensive HD on vascular access seem to be outweighed in patients with AVG and catheters by the increased frequency of cannulation and disconnection resulting in recurrent graft trauma and weakening and access infections, respectively.

Limitations of this review should be appreciated. We were not able to research the patient-specific data. Also, only a few studies were randomized controlled trials, as most studies were observational. Furthermore, the included studies varied considerably with respect to patient population; dialysis time, frequency, and location; definition of outcomes; methods of outcome assessment; follow-up times, and methodological design. This heteroge- neity is likely one factor contributing to the inconsistency of results observed. Thus, in the absence of randomized trials, several aspects of vascular access in intensive HD remain uncertain and deserve further study in future trials. The ideal vascular access type in intensive HD has yet to be defined, and may depend upon individual clinical parameters such as age, comorbidities, immunosuppressive medications, and personal preference.

In conclusion, our analysis shows an overall higher rate of vascular access complications in patients treated with intensive $\mathrm{HD}$ as compared to conventional HD. However, in patients with AVF, the event rates were relatively low both in conventional and intensive HD. Further studies are required to investigate whether AVF may be the vascular access of first choice, also for intensive HD.

\section{Acknowledgement}

We thank Qingqing Xiao from Renal Research Institute, New York, N.Y., USA, for her extensive assistance with the analytical efforts involved in this review.

\section{References}

$>1$ Uldall R, Ouwendyk M, Francoeur R, Wallace L, Sit W, Vas S, Pierratos A: Slow nocturnal home hemodialysis at the Wellesley Hospital. Adv Ren Replace Ther 1996;3:133-136.

$\checkmark 2$ Perl J, Chan CT: Home hemodialysis, daily hemodialysis, and nocturnal hemodialysis: core curriculum 2009. Am J Kidney Dis 2009; 54:1171-1184.

3 Cornelis T, Kooistra MP, Kooman JP, Leunissen KM, Chan CT, van der Sande FM: Education of ESRD patients on dialysis modality selection: 'intensive HD first'. Nephrol Dial Transplant 2010;25:129-130.

$\checkmark 4$ Woods JD, Port FK, Orzol S, Buoncristiani U, Young E, Wolfe RA, Held PJ: Clinical and biochemical correlates of starting 'daily' hemodialysis. Kidney Int 1999;55:2467-2476.

5 Quintaliani G, Buoncristiani U, Fagugli R, Kulurianu H, Ciao G, Rondini L, Lowenthal DT, Reboldi G: Survival of vascular access during daily and three times a week hemodialysis. Clin Nephrol 2000;5:372-377.

6 DePalma JR, Pecker EA, Maxwell MH: A new automatic coil dialyzer system for 'daily' dialysis. Proc Eur Dial Transplant Assoc 1969; 6:26-34.
7 Suri RS, Larive B, Sherer S, Eggers P, Gassman J, James SH, Lindsay RM, Lockridge RS, Ornt DB, Rocco MV, Ting GO, Kliger AS; Frequent Hemodialysis Network Trial Group: Risk of vascular access complications with frequent hemodialysis. J Am Soc Nephrol 2013;24: 498-505.

8 Mustafa RA, Zimmerman D, Rioux JP, Suri RS, Gangji A, Steele A, MacRae J, Pauly RP, Perkins DN, Chan CT, Copland M, Komenda P, McFarlane PA, Lindsay R, Pierratos A, Nesrallah GE: Vascular access for intensive maintenance hemodialysis: a systematic review for the Canadian Society of Nephrology Clinical Practice Guideline. Am J Kidney Dis 2013;62:112-131.

9 Pinciaroli A: Results of daily hemodialysis in Catanzaro: a 12-year experience with 22 patients treated for more than 1 year. Home Hemodial Int 1998;2:12-17.

10 Pierratos A, Ouwendyk M, Francoeur R, Vas S, Rai DSC, Ecclestone AM, Langos V, Uldall R: Nocturnal hemodialysis: three-year experience. J Am Soc Nephrol 1998;9:859-868.

11 Kjellstrand CM, Blagg CR, Twardowski ZJ, Bower J: Blood access and daily hemodialysis: clinical experience and review of the literature. ASAIO J 2003;49:645-649.
12 Lindsay R, Leitch R, Heidenheim P, Kortas C: The London Daily/Nocturnal Hemodialysis Study - study design, morbidity, and mortality results. Am J Kidney Dis 2003;42(1 suppl):5-12.

13 Ting GO, Kjellstrand C, Freitas T, Carrie BJ, Zarghamee S: Long-term study of high-comorbidity ESRD patients converted from conventional to short daily hemodialysis. Am J Kidney Dis 2003;42:1020-1035.

14 Traeger J, Galland R, Delawari E, Arkouche W, Hadden R: Six years' experience with short daily hemodialysis: do the early improvements persist in the mid and long term? Hemodial Int 2004;8:151-158.

$\checkmark 15$ Piccoli GB, Bermond F, Mezza E, Burdese M, Fop F, Mangiarotti G, Pacitti A, Maffei S, et al: Vascular access survival and morbidity on daily dialysis: a comparative analysis of home and limited care hemodialysis. Nephrol Dial Transplant 2004;19:2084-2094.

16 Williams AW, Chebrolu SB, Ing TS, Ting G, Blagg CR, Twardowski ZJ, Woredekal Y, Delano B, Gandhi VC, Kjellstrand CM: Early clinical, quality-of-life, and biochemical changes of 'daily hemodialysis' (6 dialyses per week). Am J Kidney Dis 2004;43:90-102. 
17 Reynolds JT, Homel P, Cantey L, Evans E, Harding P, Gotch F, Wuerth D, Finkelstein S, Levin N, Kliger A, Simon DB, Finkelstein FO: A one-year trial of in-center daily hemodialysis with an emphasis on quality of life. Blood Purif 2004:22:320-328.

18 Martins Castro MC, Luders C, Elias RM, Abensur H, Romao Junior JE: High-efficiency short daily haemodialysis - morbidity and mortality rate in a long-term study. Nephrol Dial Transplant 2006;21:2232-2238.

19 Perl J, Lok CE, Chan CT: Central venous catheter outcomes in nocturnal hemodialysis. Kidney Int 2006;70:1348-1354.

-20 Culleton BF, Walsh M, Klarenbach SW, Mortis G, Scott-Douglas N, Quinn RR, Tonelli M, Donnelly S, et al: Effect of frequent nocturnal hemodialysis versus conventional hemodialysis on left ventricular mass and quality of life: a randomized controlled trial. JAMA 2007; 298:1291-1299.

21 Johansen KL, Zhang R, Huang Y, Chen S-C, Blagg CR, Goldfarb-Rumyantzev AS, Hoy CD, Lockridge RS, Miller BW, et al: Survival and hospitalization among patients using nocturnal and short daily compared to conventional hemodialysis: a USRDS study. Kidney Int 2009;76:984-990.

-22 van Eps C, Jones M, Ng T, Johnson DW, Campbell SB, Isbel NM, Mudge DW, Beller E, Hawley CM: The impact of extended-hours home hemodialysis and buttonhole cannulation technique on hospitalization rates for septic events related to dialysis access. Hemodial Int 2010;14:451-463.

23 Chertow GM, Levin NW, Depner TA, Eggers PW, Gassman JJ, Gorodetskaya I, Greene T, James $\mathrm{S}$, et al: In-center hemodialysis six times per week versus three times per week. N Engl J Med 2010;363:2287-2300.
24 Rocco MV, Lockridge RS, Beck GJ, Eggers PW, Gassman JJ, Greene T, Larive B, Chan CT, Chertow GM, et al: The effects of frequent nocturnal home hemodialysis: the Frequent Hemodialysis Network Nocturnal Trial. Kidney Int 2011;80:1080-1091.

25 Achinger S, Ikizler TA, Bian A, Shintani A, Ayus JC: Long-term effects of daily hemodialysis on vascular access outcomes; a prospective controlled study. Hemodial Int 2013;17: 208-215.

26 Goldfarb-Rumyantzev AS, Leypoldt JK, Nelson N, Kutner NG, Cheung AK: A cross-over study of short daily hemodialysis. Nephrol Dial Transplant 2006;21:166-175.

27 Jun M, Jardine MJ, Gray N, Masterson R, Kerr PG, Agar JW, Hawley CM, van Eps C, Cass A, Gallagher M, Perkovic V: Outcomes of extended-hours hemodialysis performed predominantly at home. Am J Kidney Dis 2013; 61:247-253.

28 Pipkin M, Craft V, Spencer M, Lockridge RJ Jr: Six years of experience with nightly home hemodialysis access. Hemodial Int 2004; 8: 249-353.

29 NKF-K/DOQI Clinical Practice Guidelines for Vascular Access: Update 2006. Guideline 8: Clinical Outcome Goals, 8.1 Goals for Access Placement, 8.1.2 Prevalent Functional AVF Placement Rate. New York, National Kidney Foundation, 2006.

30 Allon M, Robbin ML: Increasing arteriovenous fistulas in hemodialysis patients: problems and solutions. Kidney Int 2002;62:1109.

- 31 Roy-Chaudhury P, Sukhatme VP, Cheung AK: Hemodialysis vascular access dysfunction: a cellular and molecular viewpoint. J Am Soc Nephrol 2006;17:1112-1127.

32 Weiss MF, Scivittaro V, Anderson JM: Oxidative stress and increased expression of growth factors in lesions of failed hemodialysis access. Am J Kidney Dis 2001;37:970980.
33 Roy-Chaudhury P, Kelly BS, Miller MA, Reaves A, Armstrong J, Nanayakkara N, Heffelfinger SC: Venous neointimal hyperplasia in polytetrafluoroethylene dialysis grafts. Kidney Int 2001;59:2325-2334.

-34 Stracke S, Konner K, Köstlin I, Friedl R, Jehle PM, Hombach V, Keller F, Waltenberger J: Increased expression of TGF-beta 1 and IGF-I in inflammatory stenotic lesions of hemodialysis fistulas. Kidney Int 2002;61:1011-1019.

-35 Swedberg SH, Brown BG, Sigley R, Wight TN Gordon D, Nicholls SC: Intimal fibromuscular hyperplasia at the venous anastomosis of PTFE grafts in hemodialysis patients. Clinical, immunocytochemical, light and electron microscopic assessment. Circulation 1989;80: 1726-1736.

36 Albers FJ: Causes of hemodialysis access failure. Adv Ren Replace Ther 1994;1:107-118.

-37 Chen HY, Chiu YL, Chuang YF, et al: Association of low serum fetuin A levels with poor arteriovenous access patency in patients undergoing maintenance hemodialysis. Am J Kidney Dis 2010;56:720-727.

-38 Gagliardi GM, Rossi S, Condino F, et al: Malnutrition, infection and arteriovenous fistula failure: is there a link? J Vasc Access 2010;12: 57-62.

39 Chou CY, Liu JH, Kuo HL, et al: The association between pulse pressure and vascular access thrombosis in chronic hemodialysis patients. Hypertens Res 2009;32:712-715.

40 Mallamaci F, Bonanno G, Seminara G, Rapisarda F, Fatuzzo P, Candela V, Scudo P, Spoto B, Testa A, Tripepi G, Tech S, Zoccali C: Hyperhomocysteinemia and arteriovenous fistula thrombosis in hemodialysis patients. Am J Kidney Dis 2005;45:702-707.

41 Friedman AN, Bostom AG, Levey AS, Rosenberg IH, Selhub J, Pierratos A: Plasma total homocysteine levels among patients undergoing nocturnal versus standard hemodialysis. J Am Soc Nephrol 2002;13:265-268. 